\title{
AMIZADE EM IDOSOS: um panorama da produção científica recente em periódicos estrangeiros
}

\author{
Luciana Karine de Souza ${ }^{1}$ \\ Agnaldo Garcia ${ }^{2}$
}

\section{Resumo}

O objetivo deste trabalho foi apresentar uma revisão crítica da produção científica recente sobre a amizade de idosos publicada em periódicos estrangeiros de 1989 a 2008, com base em levantamento realizado no PsycInfo. Os 65 artigos selecionados foram publicados em 44 periódicos distintos. A partir da análise dos artigos, cinco temas foram identificados: a dialética família/amigos; a amizade nas instituições de longa permanência e nos condomínios para idosos; a mulher envelhecente; apoio social, redes e tipos de amizades em idosos; e o tratamento teórico e metodológico sobre o tema da amizade em idosos. Com base na literatura científica examinada, são discutidas possibilidades para a construção de um modelo teórico para a amizade na velhice e para o avanço da pesquisa sobre o tema no Brasil. Palavras-chave: Relacionamento. Amizade. Velhice. Envelhecimento. Idoso.

\section{Introdução}

O presente texto procura dispor uma revisão recente, crítica e útil da literatura científica publicada em âmbito internacional sobre relacionamentos de amizade em idosos. Trata-se de um tema de relevância tanto científica como social. Quanto à primeira, não há estudos dedicados à amizade na velhice na mesma proporção em que há estudos sobre amizade na infância ou na adolescência, inclusive no meio científico internacional. Quanto à relevância social, o firme e compassado caminhar do envelhecimento da população brasileira e o crescimento de demandas voltadas para o público idoso requerem investimento não somente em ciências biológicas e da saúde, mas também em ciências humanas. Nesse sentido, o estudo das relações

1 Doutora em Psicologia. Professora Adjunta no Departamento de Psicologia da Universidade Federal de Minas Gerais. E-mail: luciana.karine@pq.cnpq.br

2 Doutor em Psicologia. Professor do Departamento de Psicologia Social e do Desenvolvimento e do PPGPsicologia da Universidade Federal do Espírito Santo. Bolsista de Produtividade em Pesquisa do CNPq. E-mail: agnaldo.garcia@pesquisador.cnpq.br 
interpessoais passa a focalizar a amizade na velhice para além dos estudos sobre relações sociais do idoso com familiares e/ou cuidadores.

Mediante suas características constitutivas, anteriormente mencionadas, esta revisão busca oferecer: recentidade nos trabalhos científicos, oriundos de periódicos com números publicados entre 1989 e junho de 2008; crítica destes trabalhos, classificando-se os modelos teóricos e os planos metodológicos do tratamento aos dados, quando pertinente, assim como os resultados e contribuições atingidos; e utilidade à medida que revisa uma literatura fundamentalmente publicada em língua inglesa. A este respeito cabe ressaltar que o idioma da publicação é ainda um obstáculo para uma parcela dos profissionais que trabalham na prática cotidiana com idosos nos mais distintos contextos.

A presente revisão destina-se, em especial, a complementar as leituras de indivíduos ligados a distintas áreas do conhecimento que estejam dedicando-se ao estudo do envelhecimento. Também se direciona aos profissionais educadores e cuidadores de idosos que carecem de uma atualidade científica disponível em língua portuguesa e apresentada de forma sistemática e crítica. Além do exposto, a análise pretendida das publicações encontradas sobre amizade em idosos nos periódicos estrangeiros possibilitará a visualização de um panorama atual dos modelos teóricos e do tratamento metodológico que vêm sendo utilizados nos estudos dessa natureza.

A produção científica com dados brasileiros acerca das relações sociais em idosos e, mais especificamente, sobre as relações de amizade em idosos, é ainda incipiente. De outro lado, encontram-se relatos de experiências profissionais que tratam, ainda que perifericamente, destes aspectos importantes à qualidade de vida do idoso. No entanto, é interessante observar que, na última década, o esforço científico brasileiro direcionado ao estudo da população idosa cresceu significativamente. A propósito, no Brasil atualmente conta-se com periódicos especializados em envelhecimento, a exemplo da Revista Brasileira de Ciência do Envelhecimento Humano (2004-), da Revista Estudos Interdisciplinares sobre o Envelhecimento (1999-) e da Revista Brasileira de Geriatria e Gerontologia (1998-). A busca pela produção científica brasileira sobre amizade em idosos, no entanto, foge aos propósitos do presente trabalho, mas certamente merece empenho futuro nessa direção.

Num primeiro momento, são apresentados sucintamente os conceitos de amizade mais trabalhados na literatura, e os modelos teóricos mais utilizados para organizar os dados empíricos coletados com idosos e suas amizades. A segunda parte da revisão explicita como foram coletados os trabalhos 
empíricos sobre amizade na velhice nos periódicos internacionais, e quais os critérios usados para analisá-los e organizá-los de forma sistemática e coerente. Finalmente, a partir do panorama organizado, procura-se traçar perspectivas para investigações científicas futuras, assim como sugestões de estudos a serem realizados no Brasil.

2 Relacionamentos de Amizade: da infância à velhice

A amizade vem sendo concebida de forma distinta por pesquisadores da área das ciências humanas e sociais (FEHR, 1996). De um lado, permite considerar irmãos e pais como amigos; de outro, é proposta como um relacionamento sem laços familiares ou romântico-sexuais (FEHR, 1996). Contudo, um relevante ponto a se considerar diante do espectro de definições e propostas conceituais para amizade é seu papel importante enquanto um dos três tipos de relacionamentos que mais promove satisfação de vida, bem-estar subjetivo e felicidade às pessoas, assim como os relacionamentos familiares e românticos (ARGYLE, 2001).

Estudada desde a Idade Antiga (BALDINI, 2000), a amizade pode ser classificada em tipos e/ou organizada em etapas, isto é, ela pode desenvolverse e alcançar tipos mais avançados. Num primeiro momento, os sujeitos de uma dada relação se conhecem (são conhecidos), ou são, por exemplo, colegas. Para tornarem-se amigos, precisam identificar ou um ganho instrumental com a relação, ou um ganho prazeroso (como diversão, alegria, bom-humor), ou um ganho afetivo (como confiança, lealdade). Nesse sentido, a amizade pode partir de uma relação mais generalista (de trocas instrumentais, como estudar juntos, praticar um esporte ou participar de um grupo musical) para uma amizade próxima (onde a confiança e a lealdade são recíprocas) e, por vezes, para se transformar em uma melhor amizade (FEHR, 1996).

Enquanto a amizade infantil se caracteriza essencialmente por brincadeiras, diversão e companheirismo, a amizade adolescente envolve confiança, lealdade e intimidade quanto a pensamentos e sentimentos compartilhados (BUKOWSKI; NEWCOMB; HARTUP, 1996). Na adultez jovem, o contexto universitário permite a exploração de tipos distintos de amizades e de relacionamentos românticos (RAWLINS, 1992). Na adultez com parceiro estável e/ou com filhos, a amizade perde espaço para a família e a carreira, limitando-se a poucos contatos com um número menor de amigos (CARBERY; BUHRMESTER, 1998). Na velhice, a amizade é mais heterogênea, ou seja, além de amigos de mesmo sexo, têm-se muitos amigos de sexo oposto, 
e um saudosismo quanto às amizades do passado (SHEA; THOMPSON; BLIESZNER, 1988).

Um estudo pioneiro que investigou a amizade em distintas faixas etárias do ciclo vital foi o de Weiss e Lowenthal (1975). Nesse trabalho, os autores compararam a concepção de amizade real e de amizade ideal em estudantes de ensino médio, adultos recém-casados, adultos de meia-idade e adultos maduros. Weiss e Lowenthal (1975, p. 58) observaram que "As percepções das qualidades de amigos e da amizade são surpreendentemente semelhantes através dos quatro estágios de vida." Aspectos típicos da fase da vida de cada grupo etário eram os fatores que distinguiam as amizades entre estes grupos, apontando para uma crescente seletividade nas escolhas de amizade em virtude da maturidade. Os autores concluíram que as funções básicas da amizade se estabelecem relativamente cedo com respeito à idade e permanecem ao longo da vida.

Especificamente com relação à amizade na velhice, embora seja escassa a literatura empírica, vem-se notando um crescimento desde a década de 1970, na produção científica dedicada a esta etapa da vida, no que tange especificamente a seus amigos. Uma pesquisadora de destaque é Rosemary Blieszner, que recentemente foi homenageada pela revista Personal Relationships por sua contribuição científica ao estudo dos relacionamentos interpessoais na velhice (BLIESZNER, 2006). Rebecca Adams é outra pesquisadora de destaque que, ao lado de Blieszner e de outros colaboradores, também vem produzindo de forma sistemática sobre este tema.

3 Publicações Estrangeiras sobre Amizade em Idosos: coleta e análise dos artigos

Na primeira etapa do trabalho, empreendeu-se uma busca por artigos de periódicos na base PsycInfo, considerando-se os anos de 1989 a 2008 (junho). As palavras utilizadas na busca foram friend* no título, e adult* no campo qualquer lugar (anywhere), com a expressão booleana "and". A justificativa para tais critérios está fundamentada em buscas anteriores que demonstraram resultados volumosos mediante friend* no campo qualquer lugar, encontrando muitos artigos distantes da temática alvo, qual seja, amizade em idosos. A razão pelo uso do termo adult* em vez de termos mais diretamente associados à velhice ou terceira idade também vem de buscas anteriores, nas quais se perdiam muitos trabalhos que utilizam a expressão older adults nas palavras-chave. Cabe lembrar que o uso do 
asterisco permite a busca por palavras derivadas, como adulthood, adults, friendship, friends.

A segunda etapa consistiu-se de uma leitura livre de todos os resumos encontrados, e a conseqüente exclusão de trabalhos que não tratavam da amizade em idosos, mas apenas citavam os termos da busca sem estarem relacionados. O outro critério de exclusão foi a retirada de toda e qualquer publicação que não estivesse no formato de um artigo publicado em periódico. Este critério responde à duplicação de resumos advindos de teses e dissertações, que são posteriormente publicadas em periódicos. Além disso, muitos livros e capítulos de livros não são de acesso facilitado para as análises que o presente trabalho propõe. Em complemento a isso, os pesquisadores e profissionais no Brasil têm acesso garantido a quase todos os artigos pelo site Periódicos Capes, ao passo que os livros dependem de raras verbas para as bibliotecas comprarem obras em língua estrangeira.

A terceira etapa envolveu a inclusão de artigos relacionados à temática estudada, mas que, embora não contassem na busca efetuada, foram acessados em trabalhos anteriores (ERBOLATO, 2001; GARCIA; LEONEL, 2007; SCARABELLI; GARCIA, 2006; SOUZA, 2004), dedicados exclusivamente à amizade na velhice. Ao final, foram selecionados para análise 65 artigos publicados em periódicos indexados na base de dados PsycInfo.

Freqüências foram calculadas para a quantidade de artigos por periódico. O conteúdo dos artigos foi analisado conforme o tratamento teórico e metodológico dado ao tema da amizade. Categorias temáticas foram elaboradas para cada âmbito de análise. A construção dessas categorias teve por fundamento uma adaptação da técnica de análise de conteúdo de Bardin (1977): unidades temáticas semelhantes foram identificadas nos artigos e agrupadas para formar categorias. As unidades temáticas com reduzida freqüência foram todas agrupadas como outras respostas, outros temas ou rótulos semelhantes.

\section{Resultados e Discussão}

Devido à natureza do presente trabalho, os resultados e a discussão são apresentados juntos, otimizando o aproveitamento desta última. Primeiramente são relatados os periódicos estrangeiros nos quais foram localizados os artigos selecionados. Em seguida, passa-se à análise dos 65 textos encontrados nestes periódicos.

Do total de 44 periódicos, 24 são dedicados a estudos sobre envelhecimento, sendo os demais de cunho generalista ou associados a outras 
especialidades. Dentre os periódicos que tratam especificamente da velhice, o que mais apresentou artigos contabilizou seis textos (International Journal of Aging and Human Development). Três periódicos apresentaram três artigos cada: Aging and Society, Gerontologist e Psychology and Aging. Outros sete títulos forneceram dois artigos cada, conforme a busca realizada: Activities, Adaptation and Aging; Journal of Aging Studies; Journal of Gerontological Social Work; Journals of Gerontology: Series B: Psychological Sciences and Social Sciences; Gerontology; Canadian Journal on Aging; e o Aging and Mental Health. Por terem cada um apresentado apenas um artigo, os demais periódicos podem ser conferidos na sessão de referências do presente texto.

Periódicos generalistas ou especializados em outras temáticas também foram fontes de publicação de artigos sobre amizade em idosos. Contudo, como se poderia esperar, apresentaram baixa freqüência de artigos. Os periódicos Journal of Social and Personal Relationships e o Personal Relationships indicaram três artigos cada, ao passo que o Social Work indicou dois trabalhos publicados sobre amizade em idosos entre 1989 e 2008 (junho). Os demais periódicos, não especializados em envelhecimento, apresentaram um artigo cada, e podem ser conferidos na seção de referências.

Esta diversidade de periódicos permite observar que os estudos da amizade na velhice: (a) são de natureza interdisciplinar (com contribuições provenientes da Psicologia, da Sociologia, do Serviço Social, da Gerontologia, entre outras áreas); (b) mesclam uma perspectiva sincrônica, com ênfase nas condições atuais de vida (como rede social e ambiente físico) e outra diacrônica, com ênfase nas mudanças ao longo do desenvolvimento humano; (c) a contribuição teórica é dividida com a preocupação prática, de atendimento ao idoso.

A partir destas observações, nota-se a necessidade de modelos teóricos para a amizade que atendam as diferentes abordagens consideradas. Não se pode pensar na amizade como objeto de estudo apenas da Psicologia ou da Sociologia (contempladas em alguns modelos de amizade). Tendo em vista a construção de um modelo teórico para reunir e organizar a produção sobre a amizade de idosos, devem ser levados em conta, além de fatores psicológicos e sociais, fatores fisiológicos (relacionados à saúde física do idoso) e fatores ecológicos (relacionados aos múltiplos aspectos do ambiente físico e social). Além disso, o modelo deve possibilitar a análise e a integração de fatores sincrônicos e diacrônicos. 
5 A Dialética Família/Amigos: conflito e/ou cooperação?

Uma série de estudos investiga o papel dos amigos, dos familiares e do parceiro romântico no que tange a diferentes aspectos da vida do idoso. Nesta seção, são identificadas e analisadas as investigações sobre tais aspectos.

Os artigos comparam ou analisam a influência de familiares e da amizade em distintos aspectos da vida do idoso, tendo sido identificados dezessete trabalhos. Deste total, cinco enfocam a saúde física e psicológica do idoso e o papel dos amigos e da família nessas condições, abordando temas como doença crônica (GALLANT, SPITZE; PROHASKA, 2007), deficiência visual (McILVANEA; REINHARDT, 2001), HIV/AIDS (SCHRIMSHAW; SIEGEL, 2003), depressão (MULLINS; DUGAN, 1991) e adaptação à residência em instituições de cuidado (PORT et al., 2001). Outros quatro artigos dedicaram-se a investigar a influência da família e dos amigos no bem-estar e na satisfação de vida do envelhecente (ANTONUCCI; LANSFORD; AKIYAMA, 2001; HONG; DUFF, 1997; McMULLIN; MARSHALL, 1996; SIU; PHILLIPS, 2002), e na promoção de sua saúde (SEEMAN, 2000).

Os sete últimos artigos que trataram da interação família-amigos sobre o idoso enfatizaram processos específicos intrincados nestas relações. Funções características da amizade, como apoio e companheirismo (ROOK; ITUARTE, 1999), confiança (AMAGAI, 1997), conflito (FISHER; REID; MELENDEZ, 1989) e ajuda (JOHNSON, 1998) ganharam foco nas investigações. Dois trabalhos destacaram a amizade e a família no contexto das relações intergeracionais (KEMP, 2005; O'CONNOR, 1995). Finalmente, o clássico artigo de Adams e Blieszner (1995), tratando sobre como envelhecer bem com a família e os amigos.

Os amigos, ao lado dos familiares (incluindo o cônjuge), são possivelmente as figuras mais importantes da rede social do idoso. O envelhecer, contudo, provoca mudanças nas relações familiares e nas relações com amigos quanto à estrutura da rede (lembrando que o idoso tem maior probabilidade de ter perdido um maior número de familiares e amigos) e ao papel social que desempenha. Assim, os papéis desempenhados por amigos e familiares na velhice sofrem modificações que possivelmente se afetam mutuamente (HINDE, 1997). 


\section{A Amizade nas Instituições de Cuidado e nos Condomínios para Idosos}

Como referido por Debert (1999), em alguns países economicamente mais ativos, é crescente o surgimento de condomínios fechados específicos para a população idosa. Nestes condomínios, onde o idoso é espacialmente segregado, não se preconiza a discriminação ou a separação dele por parte da família ou da sociedade. No lugar disso, pretende-se proporcionar ao idoso que prefira residir o mais independentemente possível da família uma ampliação significativa na rede de relacionamentos com pessoas que possuem a mesma faixa etária e, portanto, interesses e vivências semelhantes. Estes condomínios possuem uma equipe de saúde sempre pronta a atender o residente, sendo variada a necessidade de cuidados mais intensos ou de apenas apoio e acompanhamento profissional. Por outro lado, há também as instituições de longa permanência para idosos (p. ex., residenciais e lares), especificamente dedicadas a cuidar de idosos que necessitam de atenção intensa em saúde e cuidados diários.

$\mathrm{Na}$ presente busca de artigos sobre amizade na velhice, foram encontrados dez trabalhos no contexto dos condomínios fechados ou mesmo dos residenciais de cuidado para idosos. Dois textos tratam de amizades em contextos residenciais ou acomodações segregadas conforme a idade (DAVIDSON; BROOKE; KENDIG, 2001; McKEE; HARRISON; LEE, 1999), ao passo que outros cinco examinam as amizades na terceira idade de residentes de comunidades para aposentados (ADAY; KEHOE; FARNEY, 2006; BUYS, 2001; HONG; DUFF, 1997; JOSHI; DE-GRÂCE; BEAUPRÉ, 1989; POTTS, 1997). Também foram identificados dois artigos sobre as amizades de residentes em instituições para idosos dependentes de cuidados (BARKER, 2002; PORT et al., 2001; SHERER, 2001).

$\mathrm{O}$ ambiente físico e social, incluindo as instituições de cuidado e os condomínios para idosos, é um fator primordial no estudo de suas amizades. O ambiente físico, contudo, afeta e é afetado por estruturas sócio-culturais, de modo que o estudo do relacionamento interpessoal, em geral, e das amizades, em particular, deveria levar em conta ambos os fatores, além de sua relação dialética (HINDE, 1997).

7 A Mulher Envelhecente: amizade e saúde física, psicológica e social

Oito artigos dedicaram-se a estudar a amizade na população envelhecente do sexo feminino. Cinco textos debatem intervenções para promover 
a amizade em idosas ou ampará-las quanto a este relacionamento (ADAY; KEHOE; FARNEY, 2006; GREENBERG et al., 1999; MARTINA; STEVENS, 2006; STEVENS, 2001; STEVENS; MARTINA; WESTERHOF, 2006). Alguns destes trabalhos, inclusive, enfatizam a solidão ou o isolamento social, aspectos para os quais os programas de intervenção referidos se direcionam. Os outros três artigos sobre a amizade em idosas focalizam o bem-estar psicológico e sua interface com este relacionamento (SIU; PHILLIPS, 2002; STEVENS, 1997), um deles analisando os processos de intimidade e de confidência nos relacionamentos destas idosas (BABCHUK; ANDERSON, 1989).

Pesquisas dedicadas a idosas são recorrentes em virtude de que elas vivem mais tempo do que os idosos. Nos programas e projetos de extensão oferecidos à terceira idade no Brasil, por exemplo, é raro encontrar um equilíbrio na proporção entre homens e mulheres. Nesse sentido, é interessante notar que, no trabalho com envelhecentes, em especial no país, a escolha por métodos quantitativos para o trabalho com mulheres não pode ser repetida com a população idosa de homens. Além de se tratar de velhices distintas, ainda que possuam pontos em comum, os estudos empíricos com idosos do sexo masculino merecem tratamento metodológico diferenciado mediante técnicas qualitativas de coleta e análise dos dados.

Amizades femininas são diferentes das masculinas desde a infância. Em relação à natureza do idoso, vários aspectos sócio-demográficos devem ser ponderados para inclusão em um modelo mais amplo da amizade nesta faixa etária. Assim, questões de gênero, de etnia, de condição física, de personalidade e identidade social e cultural, entre outras, devem ser consideradas. Isto permite observar que há uma grande diversidade de idosos, do ponto de vista individual, e que esta individualidade não pode ser esquecida em um modelo teórico abrangente.

\section{Apoio Social, Redes e Tipos de Amizades em Idosos}

Nessa seção, foram destacados aqueles textos que trataram de três conjuntos de temáticas, isto é, três categorias: (1) a amizade como apoio social, redes de amizades e de contatos, e amizades próximas; (2) isolamento social, solidão, depressão e sobrevivência; e (3) amizade e bem-estar do idoso. Cabe lembrar que um mesmo artigo pode ser encontrado em distintas categorias.

No primeiro conjunto de estudos, os trabalhos de Lang e Carstensen (1994), McFadden, Knepple e Armstrong (2003), McIlvanea e Reinhardt 
(2001), Nocon e Pearson (2000), Potts (1997) e Siebert, Mutran e Reitzes (1999) dedicaram-se ao apoio social fornecido por amizades de idosos. Sete artigos analisaram as redes de amigos, quais sejam, os trabalhos de Adams e Torr (1998), Antonucci et al. (2002), Barker (2002), Bickmore et al. (2005), Dunning (2007), Pin et al. (2005) e Pinquart e Sörensen (2000). O contato com as amizades foi tema de outros quatro, os artigos de Buys (2001), Port et al. (2001), Sherer (2001) e Sugisawa et al. (2001). Tipos específicos de amizade - no caso, as amizades mais próximas - apareceram em dois estudos, de Sklar e Hartley (1990) e de Jones e Vaughan (1990).

Como se pode observar, estudos tratando de redes sociais e de relações entre membros dessa rede tipicamente tratam de estruturas e processos. Assim, as redes de amigos geralmente são tratadas de modo estrutural com uma análise concomitante dos processos psicossociais afetando o relacionamento entre os membros dessa rede, como é o caso do apoio social.

O segundo grupo de trabalhos abrangeu o isolamento social e a solidão, em oito pesquisas distintas com idosos. Trataram desses temas os artigos de Aday, Kehoe e Farney (2006), Flinday (2003), Hawthorne (2006), Joshi, De-Grâce e Beaupré (1989), Martina e Stevens (2006), Routasalo et al. (2006), Stevens (2001) e Weeks (1994). Ainda uma conseqüência comum da solidão - a depressão - foi abordada nos quatro textos de Antonucci et al. (2002), Mullins e Dugan (1991), Patterson e Bettini (1993) e Potts (1997). Por fim, De-Leon (2005) mencionou a própria sobrevivência do idoso.

Como referido nos estudos, o isolamento ainda trata da precariedade de redes sociais e da carência de relações significativas entre os membros dessa rede. Assim, estudos sobre solidão e isolamento ainda abordam as redes sociais (e suas deficiências) e dos processos psicossociais (e sua precariedade), afetando o relacionamento entre os membros dessa rede.

A última categoria que abrigou artigos sobre saúde psicológica e social do idoso reuniu os trabalhos sobre bem-estar. Cinco trabalhos foram identificados, a saber, os textos de Blieszner (1995), McKee, Harrison e Lee (1999), McMullin e Marshall (1996), Siu e Phillips (2002) e Stevens (1997), dos quais apenas um especificou o âmbito de análise do bem-estar - no caso, o âmbito psicológico (SIU; PHILLIPS, 2002).

Os estudos sobre saúde e bem-estar do idoso representam uma conjunção de fatores psicológicos, sociais, fisiológicos e ecológicos. Tais fatores deveriam ser contemplados em um modelo amplo da amizade de idosos. 


\section{O Tratamento Teórico e Metodológico da Amizade em Idosos}

Foram encontrados treze artigos que focalizam aspectos teóricos e metodológicos no trabalho científico com amizade em idosos. Quatro categorias oferecem uma organização possível para dar significado a esta produção. Num primeiro grupo de trabalhos, identificam-se aspectos como a definição da amizade na velhice (ADAMS; BLIESZNER; DEVRIES, 2000; ROBERTO; KIMBOKO, 1989), os processos envolvidos na amizade em idosos (BLIESZNER; ADAMS, 1998; FIELD, 1999; JOHNSON; TROLL, 1994), e a amizade na velhice dentro do espectro do curso de vida (ANTONUCCI; AKIYAMA, 1991; FONZI; GIANNETTI; TANI, 1997; HEYL, 2004; SHERMAN; DE-VRIES; LANSFORD, 2000).

Dois artigos discutem a relação de amizade em envelhecentes com a aplicação de modelos teóricos específicos (DUGAN; KIVETT, 1998; FINCHUM; WEBER, 2000). Outros dois trabalhos se destacam por trazer contribuições específicas para a área, como o artigo de Blieszner (2006), quando de sua recente homenagem, e a recente investigação conduzida por Heyl e Schmitt (2007), que abrange a influência de diferenças individuais e da memória de eventos pessoais passados sobre a amizade na adultez madura e na velhice. Finalmente, dois artigos se destacam por representarem novos direcionamentos na pesquisa sobre relacionamentos com amigos na terceira idade. O trabalho de Adams et al. (2006) focaliza de forma interessante as diferenças de gênero nos autores de pesquisas sobre amizade em idosos. Este tipo de trabalho, de fato, tem potencial sobre a interpretação do pesquisador sobre seus dados e o possível viés desde a criação do problema de pesquisa até a publicação dos resultados. Já o trabalho de Alley et al. (2007) apresenta relevância não apenas científica, mas também prática, à medida que se preocupa em conscientizar a população para seu envelhecimento, além de quebrar o preconceito e a discriminação contra o idoso.

Um ponto que tem caracterizado a literatura sobre a amizade é a preocupação, por parte de alguns autores, com a construção de modelos mais amplos, ultrapassando as fronteiras de uma única disciplina. Neste caso, geralmente, promovem a integração de modelos psicológicos e sociológicos. A revisão da literatura recente indica que tal abrangência ainda precisa ser ampliada. Isto significa que, além de aspectos psicológicos e sociológicos, devem ser incorporados aspectos fisiológicos e ecológicos. Como estes fatores podem ser integrados em um modelo abrangente, representa um importante objeto de pesquisa para o avanço dos estudos sobre as relações de amizade, seja de idosos, seja de qualquer outro grupo etário. 


\title{
10 Considerações Finais
}

Os estudos sobre a amizade de idosos são oriundos de diversas disciplinas e representam diferentes abordagens teóricas. De modo geral, também apresentam um caráter eminentemente pragmático. O presente estudo se dedicou a mapear um conjunto de artigos sobre amizade em idosos, procurando pontuar possíveis lacunas e possibilidades de avanços teóricos com base na literatura recente. Estudos futuros poderiam aprofundar os esforços para a construção de modelos mais abrangentes que, neste momento, necessitam de maior integração entre as ciências biológicas e as ciências humanas e sociais.

Do ponto de vista empírico, há uma carência de pesquisas sobre amizade e velhice no Brasil. Um programa mais amplo de investigação do tema deveria abordar aspectos fisiológicos (incluindo a condição física do idoso), ecológicos (incluindo aspectos do ambiente físico, dos recursos disponíveis, incluindo alimentos e espaço, além de outros aspectos econômicos), psicológicos e sociais, em suas dimensões sincrônicas e diacrônicas. É possível que tal postura consiga verdadeiramente aproximar-se da amizade e melhor estudá-la, no caso, em idosos.

\section{FRIENDSHIP IN OLDER ADULTS: an international review}

\begin{abstract}
The purpose of this work was to critically review a series of papers published in scholarly foreign journals on friendship of older adults from 1989 to 2008 and indexed in PsycInfo bibliographic database. Sixty-five selected articles were published in 44 different periodicals. Based on the papers' content, five themes were identified: family/friends dialectics; friendship in nursing homes and retirement communities; the aging woman; social support networks and types of friendship among the elderly; and theoretical and methodological issues on older adults' friendships. Based on the examined scientific literature, the authors discuss the development of a theoretical model of friendship in old age and possibilities for the advancement of research on the subject in Brazil.
\end{abstract}

Keywords: Relationship. Friendship. Older adults. Aging. Elderly. 


\section{REFERÊNCIAS}

ADAMS, Rebecca; BLIESZNER, Rosemary. Aging Well With Friends and Family. American Behavioral Scientist, Beverly Hills, v. 39, n. 2, p. 209-224, 1995.

ADAMS, Rebecca; BLIESZNER, Rosemary; DEVRIES, Brian. Definitions of Friendship in the Third Age: age, gender, and study location effects. Journal of Aging Studies, New York, v. 14, n. 1, p. 117-133, 2000.

ADAMS, Rebecca; TORR, Rachel. Factors Underlying the Structure of Older Adult Friendship Networks. Social Networks, Amsterdam, v. 20, n. 1, p. 51-61, 1998.

ADAMS, Rebecca et al. Gender-of-Author Differences in Study Design of Older Adult Friendship Surveys. Personal Relationships, New York, v. 13, n. 4, p. 503-520, 2006.

ADAY, Ronald; KEHOE, Gayle; FARNEY, Lori. Impact of Senior Center Friendships on Aging Women Who Live Alone. Journal of Women \& Aging, Binghamton, v. 18, n. 1, p. $57-73,2006$.

ALLEY, Dawn et al. Creating Elder-Friendly Communities: preparations for an aging society. Journal of Gerontological Social Work, London, v. 49, n. 1-2, p. 1-18, 2007.

AMAGAI, Yumiko. A Study on the Development of Trust in Adults and Elderly Individuals: effects of supportive feelings by families and friends. Japanese Journal of Educational Psychology, Tokyo, v. 45, n. 1, p. 79-86, 1997.

ANTONUCCI, Toni; AKIYAMA, Hiroko. Social Relationships and Aging Well. Generations, San Francisco, v. 15, n. 1, p. 39-44, 1991.

ANTONUCCI, Toni; LANSFORD, Jennifer; AKIYAMA, Hiroko. Impact of Positive and Negative Aspects of Marital Relationships and Friendships on Well-Being of Older Adults. Applied Developmental Science, Mahwah, v. 5, n. 2, p. 68-75, 2001.

ANTONUCCI, Toni et al. Differences Between Men and Women in Social Relations, Resource Deficits, and Depressive Symptomatology During Later Life in Four Nations. Journal of Social Issues, Malden, v. 58, n. 4, p. 767-783, 2002.

ARGYLE, Michael. The Psychology of Happiness. 2nd ed. New York: Taylor \& Francis, 2001.

BABCHUK, Nicholas; ANDERSON, Trudy B. Older Widows and Married Women: their intimates and confidants. International Journal of Aging and Human Development, Farmingdale, v. 28, n. 1, p. 21-35, 1989.

BALDINI, Massimo (Org.). Amizade \& Filósofos. Bauru: EDUSC, 2000.

BARDIN, Laurence. Análise de Conteúdo. Lisboa: Edições 70, 1977. 
BARKER, Judith. Neighbors, Friends, and Other Nonkin Caregivers of Community-Living Dependent Elders. Journals of Gerontology: Series B: Psychological Sciences and Social Sciences, Washington, v. 57B, n. 3, p. S158-S167, May 2002.

BICKMORE, Timothy et al. It's Just Like you Talk to a Friend: relational agents for older adults. Interacting With Computers, London, v. 17, n. 6, p. 711-735, 2005.

BLIESZNER, Rosemary. A Lifetime of Caring: dimensions and dynamics in late-life close relationships. Personal Relationships, New York, v. 13, n. 1, p. 1-18, 2006.

BLIESZNER, Rosemary. Friendship Processes and Well-Being in the Later Years of Life: implications for interventions. Journal of Geriatric Psychiatry, New York, v. 28, n. 2, p. 165-182, 1995.

BLIESZNER, Rosemary; ADAMS, Rebecca. Problems With Friends in Old Age. Journal of Aging Studies, New York, v. 12, n. 3, p. 223-238, 1998.

BUKOWSKI, William M.; NEWCOMB, Andrew F.; HARTUP, Willard W. (Ed.). Friendship and its Significance in Childhood and Adolescence: introduction and comment. In: . The Company They Keep: friendship in childhood and adolescence. Cambridge: University Press, 1996. P. 1-15.

BUYS, Laurie. Life in a Retirement Village: implications for contact with community and village friends. Gerontology, Basel, v. 47, n. 1, p. 55-59, Jan./Feb. 2001.

CARBERY, Julie; BUHRMESTER, Duane. Friendship and Need Fulfillment During Three Phases of Young Adulthood. Journal of Social and Personal Relationships, London, v. 15, n. 3, p. 393-409, 1998.

DAVIDSON, Sandra; BROOKE, Elizabeth; KENDIG, Hal. Age-Segregated Housing and Friendship Interaction for Older People. Journal of Housing for the Elderly, New York, v. 14, n. $1 / 2$, p. $123-135,2001$.

DEBERT, Guita. A Reinvenção da Velhice: socialização e processos de reprivatização do envelhecimento. São Paulo: EDUSP, 1999.

DE-LEON, Carlos. Why do Friendships Matter for Survival? Journal of Epidemiology and Community Health, London, v. 59, n. 7, p. 538-539, July 2005.

DUGAN, Elizabeth; KIVETT, Vira. Implementing the Adams and Blieszner Conceptual Model: predicting interactive friendship processes of older adults. Journal of Social and Personal Relationships, New York, v. 15, n. 5, p. 607-622, 1998.

DUNNING, Troy. Aging, Activities and the Internet: senior adult friendly search engines. Activities, Adaptation and Aging, New York, v. 31, n. 3, p. 61-62, 2007.

ERBOLATO, Regina Maria Prado Leite. Contatos Sociais: relações de amizade em três momentos da vida adulta. 2001. 294 f. Tese (Doutorado) - Instituto de Psicologia e Fonoaudiologia, Pontifícia Universidade Católica de Campinas, Campinas, 2001.

FEHR, Beverley. Friendship Processes. London: Sage, 1996. 
FIELD, Dorothy. Continuity and Change in Friendships in Advanced Old Age: findings from the berkeley older generation study. International Journal on Aging and Human Development, Farmingdale, v. 48, n. 4, p. 325-346, 1999.

FINCHUM, Tanya; WEBER, Joseph. Applying Continuity Theory to Elder Adult Friendships. Journal of Aging and Identity, New York, v. 5, n. 3, p. 159-168, 2000.

FISHER, Celia; REID, James; MELENDEZ, Marjorie. Conflict in Families and Friendships of Later Life. Family Relations, Oxford, v. 38, n. 1, p. 83-89, 1989.

FLINDAY, Robyn. Interventions to Reduce Social Isolation Amongst Older People: where is the evidence? Aging \& Society, Cambridge, v. 23, n. 5, p. 647-658, 2003.

FONZI, Ada; GIANNETTI, Enrichetta; TANI, Franca. Il Legame di Amicizia Nell'Arco della Vita: giovani e anziani a confronto. Archivio di Psicologia, Neurologie e Psichiatria, Milano, v. 58, n. 2-3, p. 133-144, 1997.

GALLANT, Mary; SPITZE, Glenna; PROHASKA, Thomas. Help or Hindrance? How family and friends influence chronic illness self-management among older adults. Research on Aging, Thousand Oaks, v. 29, n. 5, p. 375-409, 2007.

GARCIA, Agnaldo; LEONEL, Sandra B. Relacionamento Interpessoal e Terceira Idade: a mudança percebida nos relacionamentos com a participação em programas sociais para a terceira idade. Pesquisas e Práticas Psicossociais, São João del-Rei, v. 2, n. 1, p. 130-139, 2007.

GREENBERG, Sarah et al. Friendship Across the Life Cycle: a support group for older women. Journal of Gerontological Social Work, New York, v. 32, n. 4, p. 7-23, 1999.

HAWTHORNE, Graeme. Measuring Social Isolation in Older Adults: development and initial validation of the friendship scale. Social Indicators Research, Dordrecht, v. 77, n. 3, p. 521-548, 2006.

HEYL, Vera. Freundschaften im Mittleren und Hoheren Erwachsenenalter: der lange arm frühkindlicher Erfahrungen. Zeitschrift fur Gerontologie und Geriatrie, Darmstadt, v. 37, n. 5, p. 357-359, 2004.

HEYL, Vera; SCHMITT, Marina. The Contribution of Adult Personality and Recalled Parent-Child Relations to Friendships in Middle and Old Age. International Journal of Behavioral Development, London, v. 31, n. 1, p. 38-48, 2007.

HINDE, Robert. Relationships: a dialectical perspective. Hove: Psychology Press, 1997.

HONG, Lawrence; DUFF, Robert. Relative Importance of Spouses, Children, and Friends in the Life Satisfaction of Retirement Community Residents. Journal of Clinical Geropsychology, New York, v. 3, n. 4, p. 275-282, 1997.

JOHNSON, Charles; TROLL, Lilian. Constraints and Facilitators to Friendships in Late Life. Gerontologist, Washington, v. 34, n. 1, p. 79-87, 1994. 
JOHNSON, Julie. Older Rural Adults and the Decision to Stop Driving: the influence of family and friends. Journal of Community Health Nursing, Hillsdale, v. 15, n. 4, p. 205-216, 1998.

JONES, Diane; VAUGHAN, Kristen. Close Friendships Among Senior Adults. Psychology and Aging, Arlington, v. 5, n. 3, p. 451-457, 1990.

JOSHI, Purushottam; DE GRÂCE, G.; BEAUPRÉ, C. Nature des contacts avec les amis et solitude chez les personnes âgées vivant dans la communauté. Cahiers Internationaux de Psychologie Sociale, Louveigné, n. 4, p. 11-25, 1989.

KEMP, Candace. Dimensions of Grandparent-Adult Grandchild Relationships: from family ties to intergenerational friendships. Canadian Journal on Aging, Maple, v. 24, n. 2, p. 161-178, 2005.

LANG, Frieder; CARSTENSEN, Laura. Close Emotional Relationships in Late Life: further support for proactive aging in the social domain. Psychology and Aging, Washington, v. 9, n. 2, p. 315-324, 1994.

MARTINA, Camille; STEVENS, Nan. Breaking the Cycle of Loneliness? Psychological Effects of a Friendship Enrichment Program for Older Women. Aging \& Mental Health, Abingdon, v. 10, n. 5, p. 467-475, 2006.

McFADDEN, Susan; KNEPPLE, Amy; ARMSTRONG, Jessica. Length and Locus of Friendship Influence Church Members' Sense of Social Support and Comfort with Sharing Emotions. Journal of Religious Gerontology, New York, v. 15, n. 4, p. 39-55, 2003.

McILVANEA, Jessica; REINHARDT, Joann. Interactive Effect of Support From Family and Friends in Visually Impaired Elders. Journals of Gerontology B: Psychological Sciences and Social Sciences, Washington, v. 56, n. 6, p. 374-382, 2001.

McKEE, K.; HARRISON, G.; LEE, K. Activity, friendships and well-being in residential settings for older people. Aging \& Mental Health, Abingdon, v. 3, n. 2, p. 143-152, 1999.

McMULLIN, Julie; MARSHALL, Victor. Family, Friends, Stress, and Well-Being: does childlessness make a difference? Canadian Journal on Aging, Maple, v. 15, n. 3, p. 355-373, 1996.

MULLINS, Larry; DUGAN, Elizabeth. Elderly Social Relationships with Adult Children and Close Friends and Depression. Journal of Social Behavior and Personality, San Rafael, v. 6 , n. 2, p. 315-328, 1991.

NOCON, Andrew; PEARSON, Maggie. The roles of friends and neighbours in providing support for older people. Aging \& Society, Cambridge, v. 20, n. 3, p. 341-367, 2000.

O'CONNOR, Brian. Family and Friend Relationships Among Older and Younger Adults: interaction motivation, mood, and quality. International Journal of Aging and Human Development, Farmingdale, v. 40, n. 1, p. 9-29, 1995. 
PATTERSON, Brian; BETTINI, Lorraine. Age, Depression, and Friendship: development of a general friendship inventory. Communication Research Reports, Morgantown, v. 10, n. 2 , p. $161-170,1993$.

PIN, Stéphanie et al. The Impact of Social Relationships on the Maintenance of Independence in Advanced Old Age: findings of a swiss longitudinal study. Zeitschrift für Gerontologie und Geriatrie, Darmstadt, v. 38, n. 3, p. 203-209, 2005.

PINQUART, Martin; SÖRENSEN, Silvia. Influences of Socioeconomic Status, Social Network and Competence on Subjective Well-Being in Later Life: a meta-analysis. Psychology and Aging, Arlington, v. 15, n. 2, p. 187-224, June 2000.

PORT, Cynthia et al. Resident Contact with Family and Friends Following Nursing Home Admission. Gerontologist, Washington, v. 41, n. 5, p. 589-596, Oct. 2001.

POTTS, Marilyn. Social Support and Depression Among Older Adults Living Alone: the importance of friends within and outside of a retirement community. Social Work, New York, v. 42, n. 4, p. 348-362, July 1997.

RAWLINS, William K. Friendship Matters: communication, dialectics, and the life course. New York: Aldine de Gruyter, 1992.

ROBERTO, Karen A.; KIMBOKO, Priscilla J. Friendships in Later Life: definitions and maintenance patterns. International Journal of Aging and Human Development, Farmingdale, v. 28, n. 1, p. 9-19, 1989.

ROOK, Karen; ITUARTE, Philip. Social Control, Social Support, and Companionship in Older Adults' Family Relationships and Friendships. Personal Relationships, New York, v. 6, n. 2, p. 199-211, 1999.

ROUTASALO, Pirkko et al. Social Contacts and their Relationship to Loneliness among Aged People: a population-based study. Gerontology, Basel, v. 52, n. 3, p. 181-187, 2006.

SCARABELLI, Rodrigo; GARCIA, Agnaldo. As Amizades dos Idosos Vivendo em Ambiente Familiar e dos Idosos Vivendo em Instituições: uma análise comparativa. In: GARCIA, Agnaldo (Org.). Relacionamento Interpessoal: estudos brasileiros. Vitória: Núcleo Interdisciplinar para o Estudo do Relacionamento Interpessoal, 2006. P. 105-121.

SCHRIMSHAW, Eric; SIEGEL, Karolynn. Perceived Barriers to Social Support From Family and Friends Among Older Adults With HIV/AIDS. Journal of Health Psychology, London, v. 8, n. 6, p. 738-752, Nov. 2003.

SEEMAN, Teresa. Health Promoting Effects of Friends and Family on Health Outcomes in Older Adults. American Journal of Health Promotion, Lawrence, v. 14, n. 6, p. 362-370, July/Aug. 2000.

SHEA, Laurie; THOMPSON, Linda; BLIESZNER, Rosemary. Resources in Older Adults' Old and New Friendships. Journal of Social and Personal Relationships, London, v. 5, p. 83-96, 1988. 
SHERER, Moshe. Interactions With Friends in a Nursing Home and Residents' Morale. Activities, Adaptation \& Aging, New York, v. 26, n. 1, p. 23-40, 2001.

SHERMAN, Aurora; DE-VRIES, Brian; LANSFORD, Jennifer. Friendship in Childhood and Adulthood: lessons across the life span. International Journal of Aging and Human Development, Farmingdale, v. 51, n. 1, p. 31-51, 2000.

SIEBERT, Darcy; MUTRAN, Elizabeth; REITZES, Donald. Friendship and Social Support: the importance of role identity to aging adults. Social Work, New York, v. 44, n. 6 , p. 522-533, 1999.

SIU, Oi-Ling; PHILLIPS, David. A Study of Family Support, Friendship, and Psychological Well-Being Among Older Women in Hong Kong. International Journal of Aging and Human Development, Farmingdale, v. 55, n. 4, p. 299-319, 2002.

SKLAR, Fred; HARTLEY, Shirley. Close Friends as Survivors: bereavement patterns in a "hidden" population. Omega: Journal of Death and Dying, Formingdale, v. 21, n. 2, p. 103-112, 1990.

SOUZA, Luciana Karine de. Velho Amigo, Amigo Velho: amizade na velhice. In: CASTRO, Odair Perugini de (Org.). Envelhecer: revisitando o corpo. Sapucaia do Sul: Notadez, 2004. P. 69-86.

STEVENS, Nan. Combating Loneliness: a friendship enrichment programme for older women. Aging \& Society, Cambridge, v. 21, n. 2, p. 183-202, 2001.

STEVENS, Nan. Vriendschap Als Sleutel Tot Welbevinden: een cursus vor vrouwen boven de 55 jahr. Tijdschrift voor Gerontologie en Geriatrie, Deventer, v. 28, n. 1, p. 18-26, 1997.

STEVENS, Nan; MARTINA, Camille; WESTERHOF, Gerben. Meeting the Need to Belong: predicting effects of a friendship enrichment program for older women. Gerontologist, Washington, v. 46, n. 4, p. 495-502, 2006.

SUGISAWA, Hidehiro et al. What Characteristics Have the Elderly Who Contact Friends at Hospitals? Nippon Koshu Eisei Zasshi, Tokyo, v. 48, n. 10, p. 807-815, 2001.

WEEKS, David. A Review of Loneliness Concepts, With Particular Reference to Old Age. International Journal of Geriatric Psychiatry, Chichester, v. 9, n. 5, p. 345-355, 1994.

WEISS, Lawrence; LOWENTHAL, Marjorie Fiske. Life-Course Perspectives on Friendship. In: LOWENTHAL, Marjorie Fiske; THURNHER, Majda; CHIRIBOGA, David (Org.). Four Stages of Life: a comparative study of women and men facing transitions. San Francisco: Jossey-Bass, 1975. P. 48-61.

Recebido em: 06-09-2008

$1^{a}$ revisão: $23-10-2008$

Aceite final: $30-10-2008$ 